TRANSACTIONS OF THE

AMERICAN MATHEMATICAL SOCIETY

Volume 351, Number 2, February 1999, Pages 477-495

S 0002-9947(99)02406-X

\title{
GLOBAL CHARACTER FORMULAE FOR COMPACT LIE GROUPS
}

\author{
A. H. DOOLEY AND N. J. WILDBERGER
}

\begin{abstract}
We introduce the concept of a modulator, which leads to a family of character formulae, each generalizing the Kirillov formula. For a suitable choice of modulator, this enables one to understand the Plancherel measure of a compact Lie group as arising from a partition of the identity on the dual of its Lie algebra.
\end{abstract}

\section{INTRODUCTION}

In a recent article [1], the authors introduced the wrapping map $\Phi$, defined for a distribution on the Lie algebra $\mathfrak{g}$ of a compact Lie group $G$ by

$$
\langle\Phi \mu, f\rangle=\left\langle\mu, j f^{\sim}\right\rangle
$$

where $f \in C^{\infty}(G), f^{\sim}=f \circ \exp$ and $j$ is an analytic square root of the determinant of exp. (Actually, some restrictions need to be placed on $\mu$ in order for this to be defined-for example it is well-defined if $\mu$ has compact support, or if $j \mu \in L^{1}(\mathfrak{g})$.) Our principal result was that $\Phi$ is a homomorphism from the algebra of Ad-invariant distributions of compact support on $\mathfrak{g}$ with Euclidean convolution to the algebra of central distributions on $G$ with the convolution structure from $G$.

The purpose of this article is to examine the algebraic structure of the Adinvariant distributions on $\mathfrak{g}$ induced by the map $\Phi$. We will show for example that the algebra of Ad-invariant functions in $L^{1}(\mathfrak{g})$ may be decomposed as a direct sum of the kernel $\mathcal{K}$ of $\Phi$ and a family $\left\{\mathcal{M}_{\lambda}: \lambda \in \Lambda^{+}\right\}$of ideals indexed by the highest weights of $G$. In fact, $\mathcal{M}_{\lambda}$ consists of those elements whose wrap is the irreducible character $\chi_{\lambda}$ associated to $\lambda$, and this leads naturally to consideration of character formulae.

Of particular interest in this context is the space $\mathcal{M}=\mathcal{M}_{0}$ of modulators of $G$, the space of distributions whose wrap is the constant function 1. It turns out that for each $m \in \mathcal{M}$ there is a character formula for $G$ which generalizes the classical Kirillov formula. The formulae obtained in this way have the form

$$
\left\langle\chi_{\lambda}, f\right\rangle=\left\langle C h_{\lambda}^{m}, j f^{\sim}\right\rangle
$$

where $C h_{\lambda}^{m}$ is a distribution on $\mathfrak{g}$. The formulae utilize the whole lift of $f$ to the Lie algebra, not just its restriction to a neighbourhood of zero. Furthermore, one may choose $m$ in such a way that $C h_{\lambda}^{m}$ has certain desirable properties of smoothness, integrability, or integrability of the Fourier transform.

Received by the editors April 30, 1995

1991 Mathematics Subject Classification. Primary 22E30, Secondary 43A75.

(C)1999 American Mathematical Society 
Indeed, when the Fourier transform of $C h_{\lambda}^{m}$ is defined, it has the form $\widehat{M} * \mu_{\lambda+\delta}$, where $\mu_{\lambda+\delta}$ is the invariant measure on the coadjoint orbit through $\lambda+\delta$. Here, as usual, $\delta$ is the half sum of the positive roots. Thus, the character formula we produce may be seen as replacing the coadjoint orbit by a "fattened" coadjoint orbit, or replacing the Dirac measure on the orbit by an "electron cloud" density centred on the orbit.

In the case of nilpotent Lie groups, it is usual to construct Plancherel measure on the dual by taking the quotient of Lebesgue measure on $\mathfrak{g}^{*}$ under the coadjoint action. This is explained in [9]. (Of course, $j$ is trivial in this case.) One usually thinks of the compact case as being at the opposite end of the spectrum - the orbits are discrete and there is not an obvious way to decompose Lebesgue measure on $\mathfrak{g}^{*}$ to obtain Plancherel measure. Using modulators, we are able to do this. Specifically, we find a particular modulator $m$, which is a version of the Fejér kernel for compact groups, and a locally finite partition of unity in $\mathfrak{g}^{*}$ of the form $\left\{d_{\lambda} F_{\lambda}: \lambda \in \Lambda^{+}\right\} \cup K$ consisting of functions of compact support, so that $F_{\lambda}=\left(C h_{\lambda}^{m}\right)^{\vee}$ and $K \subset \mathcal{K}^{\vee}$. Thus, Lebesgue measure on $\mathfrak{g}^{*}$ is decomposed into certain parts whose Fourier transforms wrap to zero and other parts whose Fourier transforms wrap exactly to the functions $d_{\lambda} \chi_{\lambda}$. This can furthermore be done in such a way that $C h_{\lambda}^{m}$ is in $L^{1}(\mathfrak{g}) \cap C^{\infty}(\mathfrak{g})$.

Apart from its intrinsic interest as a unification of the compact and nilpotent cases, we believe that this will have applications in analysis on compact Lie groups.

The plan of the paper is as follows. Section 2 contains the general functional analytic picture. Section 3 reduces the problem of finding modulators on $G$ to that of finding modulators on the maximal torus - this is done by the Poisson summation formula. Section 4 shows how to choose a particular modulator which decomposes Lebesgue measure on $\mathfrak{g}^{*}$ to obtain Plancherel measure on $\widehat{G}$. Section 5 extends the notion of modulator to certain distributions defined by principal-valued integrals.

The wrapping map has been used in Fourier analysis of central functions on compact Lie groups ([5], [8]). The results of this paper may similarly be applied.

An earlier version of this paper was sent to M. Vergne. Her comments were extremely valuable and have had a considerable impact on the current version. We would like to acknowledge the support of the Australia Research Council.

\section{Modulators}

Notation 2.1. We will use notation from [3] and [4]; this is almost standard for compact Lie groups, but let us recall some of the more important definitions, normalizations, etc.

Let $G$ be a compact connected Lie group with Lie algebra $\mathfrak{g}$. Let $T$ be a maximal torus of $G$ and $\mathfrak{t}$ its Lie algebra. Denote by $\Phi_{+}$a choice of positive roots of $(\mathfrak{g}, \mathfrak{t})$ relative to a choice of positive Weyl chamber $\mathfrak{t}_{+}$in $\mathfrak{t}$. Since roots are purely imaginary-valued, we set $R_{+}=\left\{\alpha \in \mathfrak{t}^{*}: i \alpha \in \Phi_{+}\right\}$. The Ad-invariant function $j$, defined on $\mathfrak{g}$ by

$$
j(H)=\prod_{\alpha \in R_{+}} \frac{\sin \alpha(H)}{\alpha(H)} \quad \forall H \in \mathfrak{t},
$$

is then a real-valued analytic square root of the determinant of the exponential map with $j(0)=1$. 
We normalise Haar measures $d g$ on $G$ and $d t$ on $T$ to have total mass 1. Lebesgue measure $d X$ on $\mathfrak{g}$ is normalised so that if $U$ is a neighbourhood of 0 in $\mathfrak{g}$ on which the exponential map is injective, then for $f \in C^{\infty}(G)$,

$$
\int_{U} f(\exp X)|j(X)|^{2} d X=\int_{\exp U} f(g) d g .
$$

Similarly, Lebesgue measure $d H$ on $\mathfrak{t}$ is normalised so that if $V$ is a neighbourhood of 0 in $\mathfrak{t}$ on which the exponential map is injective, then for $f \in C^{\infty}(T)$

$$
\int_{V} f(\exp H) d H=\int_{\exp V} f(t) d t .
$$

For a measurable function $\varphi$ on $\mathfrak{g}$, let $\varphi^{G}(X)=\int_{G} \varphi(g X) d g$, where $g X$ means $\operatorname{Ad}(g) X$. One then has the Weyl integration formula on $\mathfrak{g}$,

$$
\int_{\mathfrak{g}} \varphi(X) d X=\int_{\mathfrak{t}_{+}} \prod_{\alpha \in R_{+}} \alpha(H)^{2} \varphi^{G}(H) d H
$$

Similarly, for a function $f$ on $G$, define $f^{G}\left(g^{\prime}\right)=\int_{G} f\left(g g^{\prime} g^{-1}\right) d g$. The Weyl integration formula on $G$ takes the form

$$
\int_{G} f(g) d g=\frac{1}{|W|} \int_{T}|\Delta(t)|^{2} f^{G}(t) d t,
$$

where $\Delta(\exp H)=j(H) \prod_{\alpha \in R_{+}} \alpha(H)$.

Every irreducible representation $\pi \in \widehat{G}$ is associated with a unique highest weight $\lambda \in \Lambda_{+}$, where, as usual, $\Lambda \subseteq \mathfrak{t}^{*}$ denotes the set of integral weights and $\Lambda_{+}$the dominant integral weights. If $\chi_{\lambda}$ is the character of this representation, then Kirillov's character formula is

$$
j(X) \chi_{\lambda}^{\sim}(X)=\int_{\mathcal{O}_{\lambda+\delta}} e^{i \beta(X)} d \mu_{\lambda+\delta}(\beta) \quad \forall X \in \mathfrak{g},
$$

where $\mathcal{O}_{\lambda+\delta}$ is the coadjoint orbit throught $\lambda+\delta \in \mathfrak{t}^{*} \subset \mathfrak{g}^{*}$ (this inclusion is defined by the Killing form on the semisimple part) and $\mu_{\lambda+\delta}$ is Liouville measure on $\mathcal{O}_{\lambda+\delta}$; that is, the unique $G$-invariant measure with total mass $d_{\lambda}=\operatorname{dim} \pi$. Here $\delta=\frac{1}{2} \sum_{\alpha \in R_{+}} \alpha$.

With these normalizations, one may calculate the Fourier transform of an orbit by Harish-Chandra's formula,

$$
\int_{\mathcal{O}_{\lambda}} e^{i \beta(H)} d \mu_{\lambda}(\beta)=\frac{A(\lambda)(H)}{\prod_{\alpha \in R_{+}} i \alpha(H)} \quad \forall H \in \mathfrak{t}_{+}, \forall \lambda \in \mathfrak{t}_{+}^{*},
$$

where $A(\lambda)(H)=\sum_{w \in W} \operatorname{sgn} w e^{i w \lambda(H)}$, and where we interpret the right hand side by using l'Hôpital's rule in $H$.

This formula actually extends to all of $t^{*}$ by use of l'Hôpital's rule in $\lambda$. We let $\mu_{\lambda}$ be Liouville measure (see [1]), that is, $\mu_{\lambda}$ is the $G$-invariant measure on $\mathcal{O}_{\lambda}$ normalized to have total mass $\prod_{\alpha \in R_{+}}\langle\alpha, \lambda\rangle$. As usual, $\langle\alpha, \lambda\rangle=\frac{2(\alpha, \lambda)}{(\alpha, \alpha)}$, where $\langle\alpha, \lambda\rangle \neq 0$ 
( , ) is the Killing form. As $\lambda$ approaches the walls of $\mathfrak{t}_{+}$, the right hand side of Harish-Chandra's formula approaches

$$
\frac{\sum_{\{w \in W: w \lambda \neq \lambda\}} \operatorname{sgn} w e^{i w \lambda(H)}}{\prod_{\left\{\alpha \in R_{+}:\langle\alpha, \lambda\rangle \neq 0\right\}}\langle\alpha, \lambda\rangle \prod_{\alpha \in R_{+}} \alpha(H)},
$$

and the left hand side approaches the Fourier transform of Liouville measure.

We also have a version of the "dual" Weyl integration formula for $\mathfrak{g}^{*}$, in terms of the canonical Liouville measures $\mu_{\lambda}$; for a function $\varphi \in L^{1}\left(\mathfrak{g}^{*}\right)$, one defines $\varphi^{G}(\lambda)=\int_{\mathcal{O}} \varphi(\beta) d \mu_{\lambda}(\beta)$. Note that as opposed to the adjoint case, this is not a $G$-average. This reflects the fact that co-adjoint orbits carry canonical measures, but adjoint orbits do not. Then

$$
\int_{\mathfrak{g}^{*}} \varphi(\eta) d \eta=\int_{\mathfrak{t}_{+}^{*}} \varphi^{G}(\lambda) \prod_{\alpha \in R_{+}}\langle\alpha, \lambda\rangle d \lambda .
$$

Given a choice of Lebesgue measure $d \lambda$ on $\mathfrak{t}^{*}$, this defines a Lebesgue measure $d \eta$ on $\mathfrak{g}^{*}$.

We will be interested also in using the inverse Fourier transform on $\mathfrak{g}^{*}$ and $\mathfrak{t}^{*}$. Fix the Lebesgue measure $d \lambda$ on $\mathfrak{t}^{*}$ so that for $f \in C_{c}^{\infty}(\mathfrak{t})$,

$$
f(0)=\int_{\mathfrak{t}^{*}} \hat{f}(\lambda) d \lambda, \text { where } \hat{f}(\lambda)=\int_{\mathfrak{t}} f(H) e^{-i \lambda H} d H .
$$

Now consider the inverse Fourier transform on $\mathfrak{g}^{*}$. By general considerations, there is a constant $C_{G}$ such that for $\varphi \in C_{c}^{\infty}(\mathfrak{g})$,

$$
\varphi(0)=C_{G} \int_{\mathfrak{g}^{*}} \varphi^{\wedge}(\eta) d \eta, \text { where } \varphi^{\wedge}(\eta)=\int_{\mathfrak{g}} \varphi(X) e^{-i \eta X} d X .
$$

We can compute the constant $C_{G}$ as follows. Suppose that $\varphi$ is an Ad-invariant function in $C_{c}^{\infty}(\mathfrak{g})$. Then

$$
\begin{aligned}
& \varphi(0)=C_{G} \int_{\mathfrak{g}^{*}} \int_{\mathfrak{g}} \varphi(X) e^{-i \eta X} d X d \eta \\
& =C_{G} \int_{\mathfrak{t}_{+}^{*}} \int_{G} \int_{\mathfrak{g}} e^{-i g \lambda(X)} \varphi(X) d X d g \prod_{\alpha \in R_{+}}\langle\alpha, \lambda\rangle^{2} d \lambda \\
& =C_{G} \int_{\mathfrak{t}_{+}^{*}} \int_{\mathfrak{t}_{+}} \int_{G} \int_{G} e^{-i g \lambda\left(g_{1} X\right)} d g \varphi\left(g_{1} H\right) d g_{1} \prod_{\alpha \in R_{+}} \alpha(H)^{2} d H \prod_{\alpha \in R_{+}}\langle\alpha, \lambda\rangle^{2} d \lambda \\
& =C_{G} \int_{\mathfrak{t}_{+}^{*}} \int_{\mathfrak{t}_{+}} \frac{A(-\lambda)(H)}{\prod_{\alpha \in R_{+}}(-i) \alpha(H) \prod_{\alpha \in R_{+}}\langle\alpha, \lambda\rangle} \varphi(H) \prod_{\alpha \in R_{+}} \alpha(H)^{2} \prod_{\alpha \in R_{+}}\langle\alpha, \lambda\rangle^{2} d H d \lambda,
\end{aligned}
$$

where we have used Harish-Chandra's formula. Cancellation and use of the definition of $A(\lambda)(H)$ yield

$$
\varphi(0)=C_{G} \sum_{w \in W} \int_{\mathfrak{t}_{+}^{*}} \int_{\mathfrak{t}_{+}} \operatorname{sgn} w e^{-i w \lambda(H)} \varphi(H) \prod_{\alpha \in R_{+}} i \alpha(H) d H \prod_{\alpha \in R_{+}}\langle\alpha, \lambda\rangle d \lambda .
$$


Since $\prod_{\alpha \in R_{+}} \alpha$ is $W$-anti-invariant and $\left.\varphi\right|_{\mathfrak{t}}$ is $W$-invariant, this expression reduces to

$$
\begin{aligned}
\varphi(0)= & C_{G} \int_{\mathfrak{t}_{+}^{*}} \int_{\mathfrak{t}} \prod_{\alpha \in R_{+}} i \alpha(H) \varphi(H) e^{-i \lambda(H)} d H \prod_{\alpha \in R_{+}}\langle\alpha, \lambda\rangle d \lambda \\
& =C_{G} \int_{\mathfrak{t}_{+}^{*}}\left(\left.\prod_{\alpha \in R_{+}} i \alpha(H) \varphi\right|_{\mathfrak{t}}\right)^{\wedge}(\lambda) \prod_{\alpha \in R_{+}}\langle\alpha, \lambda\rangle d \lambda .
\end{aligned}
$$

The integrand, a product of two $W$-anti-invariant functions, is $W$-invariant, and thus we have

$$
\varphi(0)=\frac{C_{G}}{|W|} \int_{\mathfrak{t}^{*}} \prod_{\alpha \in R_{+}}\langle\alpha, \lambda\rangle\left(\left.\prod_{\alpha \in R_{+}} i \alpha(\cdot) \varphi\right|_{\mathfrak{t}}\right)^{\wedge}(\lambda) d \lambda .
$$

Now $\langle\alpha, \lambda\rangle=\frac{2(\alpha, \lambda)}{(\alpha, \alpha)}$, and by the elementary properties of the Fourier tranform

$$
\left.i(\alpha, \lambda) \varphi^{\wedge}(\lambda)=\left(\nabla_{\alpha}\right) \varphi\right)^{\wedge}(\lambda),
$$

where $\nabla_{\alpha}$ is the gradient of the function $\alpha$ on $\mathfrak{t}$. Thus, our expression reduces to

$$
\frac{C_{G}}{|W|} \int_{\mathfrak{t}^{*}}\left(\prod_{\alpha \in R_{+}} \frac{2}{(\alpha, \alpha)} \nabla_{\alpha}\left(\left.\prod_{\alpha \in R_{+}} \alpha(\cdot) \varphi\right|_{\mathfrak{t}}(\cdot)\right)\right)^{\wedge}(\lambda) d \lambda,
$$

and by our choice of Lebesgue measure on $\mathfrak{t}^{*}$, this equals

$$
\frac{C_{G}}{|W|} \prod_{\alpha \in R_{+}}\left(\frac{2}{(\alpha, \alpha)} \nabla_{\alpha}\right)\left(\left.\prod_{\alpha \in R_{+}} \alpha(\cdot) \varphi\right|_{\mathfrak{t}}(\cdot)\right)(0) .
$$

Since the functions $\alpha(\cdot)$ are all linear functions, Leibniz' rule shows that this expression equals

$$
\frac{C_{G}}{|W|} \prod_{\alpha \in R_{+}} \frac{2}{(\alpha, \alpha)} \nabla_{\alpha}\left(\prod_{\alpha \in R_{+}} \alpha(\cdot)\right) \varphi(0)
$$

We see that

$$
C_{G}=|W| \frac{\prod_{\alpha \in R_{+}} \frac{(\alpha, \alpha)}{2}}{\left.\prod_{\alpha \in R_{+}} \nabla_{\alpha}\left(\prod_{\alpha \in R_{+}} \alpha(\cdot)\right)\right|_{H=0}} .
$$

It is standard that if $\alpha$ and $\beta$ are linear, then $\nabla_{\alpha} \beta=(\alpha, \beta)$. From this, it follows using the Leibniz rule that

$$
\left.\prod_{\alpha \in R_{+}} \nabla_{\alpha}\left(\prod_{\alpha \in R_{+}} \alpha(\cdot)\right)\right|_{H=0}=\sum_{\sigma} \prod_{\alpha \in R_{+}}(\sigma(\alpha), \alpha),
$$

where the sum is over all permutations $\sigma$ of the positive roots. Denote by $p$ the number of positive roots, and consider the $p \times p$ matrix $\tilde{C}=(\langle\alpha, \beta\rangle)_{\alpha, \beta \in R_{+}}$; call 
this the extended Cartan matrix of the root system. By multilinearity of the permanent and the above remarks, we obtain

$$
C_{G}=\frac{|W|}{\operatorname{Perm}(\tilde{C})} .
$$

Definition 2.2. For a distribution $\mu \in \mathcal{D}^{\prime}(\mathfrak{g})$ and a function $f \in C^{\infty}(G)$, we let

$$
\langle\Phi \mu, f\rangle=\left\langle\mu, j f^{\sim}\right\rangle
$$

where $f^{\sim}(X)=f(\exp X)$, whenever the right hand side is defined.

Obviously this definition is not valid for an arbitrary distribution, as $j f^{\sim}$ will not necessarily belong to $\mathcal{D}(\mathfrak{g})$ (nor even $\mathcal{S}(\mathfrak{g})$ ). However, two circumstances in which $\Phi \mu$ is well-defined are

(a) if $\mu \in \mathcal{E}(\mathfrak{g})$ (i.e. $\mu$ has compact support on $\mathfrak{g}$ ), or

(b) if $j \mu \in L^{1}(\mathfrak{g})$.

If either of these conditions holds, we say that $\Phi \mu$, the wrap of $\mu$, is well-defined.

Actually, it turns out that since $f^{\sim}$ is in some sense periodic on $\mathfrak{g}$, one can make sense of the wrap of certain distributions on $\mathfrak{g}$ defined by principal valued integrals. We shall discuss this later $(\S 5)$.

Definition 2.3. Let

$$
\mathcal{K}=\left\{\mu \in \mathcal{D}^{\prime}(\mathfrak{g}): \Phi \mu \text { is well-defined and } \Phi \mu=0\right\}
$$

and let

$$
\mathcal{M}=\left\{m \in \mathcal{D}^{\prime}(\mathfrak{g}): \Phi m \text { is well-defined and } \Phi m=1\right\} .
$$

Elements of $\mathcal{M}$ are called $G$-modulators.

We give some basic properties of $\mathcal{K}$ and $\mathcal{M}$. If $A$ is a space of functions or distributions on $\mathfrak{g}$ or on $G$, we let $A_{G}$ denote the subspace of Ad-invariant or central elements.

Proposition 2.4. (i) If $j \mu \in L_{G}^{1}(\mathfrak{g})$ or $\mathcal{E}_{G}(\mathfrak{g})$, then $\mu \in \mathcal{K}$ if and only if $\hat{\mu}(\lambda)=0$ for all $\lambda \in \Lambda$.

(ii) Let $m \in \mathcal{M}$ and $f \in C^{\infty}(G)$. Then $\Phi\left(m f^{\sim}\right)$ is well-defined and $\Phi\left(m f^{\sim}\right)=f$. In particular $\Phi\left(m \chi_{\lambda}^{\sim}\right)=\chi_{\lambda}$.

(iii) Let $\mathcal{M}_{\lambda}=\left\{m \in \mathcal{D}^{\prime}(\mathfrak{g}): \Phi m\right.$ is well-defined and $\left.\Phi m=\chi_{\lambda}\right\}$ Then $\mathcal{M}_{0}=\mathcal{M}$ and $\mathcal{M}_{\lambda}=\chi_{\lambda}^{\sim} \mathcal{M}$.

Proof. i) If $\mu \in \mathcal{D}^{\prime}$ is $G$-invariant, then $\Phi \mu$ is a central distribution on $G$; thus $\Phi \mu=0$ if and only if $\left\langle\Phi \mu, \chi_{\lambda}\right\rangle=0 \forall \lambda \in \Lambda$. But from Kirillov's character formula, $\left\langle\Phi \mu, \chi_{\lambda}\right\rangle=\left\langle\mu, e^{-i \lambda}\right\rangle=\hat{\mu}(\lambda)$.

(ii) Let $m \in \mathcal{M}$ and $f \in C^{\infty}(G)$. Then for any $h \in C^{\infty}(G)$,

$$
\langle\Phi(m \tilde{f}), h\rangle=\langle m \tilde{f}, j \tilde{h}\rangle=\langle m, j \tilde{f} \tilde{h}\rangle=\langle\Phi m, f h\rangle=\int_{G} f(g) h(g) d g
$$

so $\Phi(m \tilde{f})=f$.

(iii) $\mathcal{M}_{0}=\mathcal{M}$ is obvious since $\chi_{0} \equiv 1$. The rest follows from (ii).

Using the sets $\mathcal{M}_{\lambda}$, we may decompose $L_{G}^{1}(\mathfrak{g})$ as an algebra.

Proposition 2.5. For each $\lambda \in \Lambda_{+}, \mathbb{C} \mathcal{M}_{\lambda} \cap L_{G}^{1}(\mathfrak{g})$ is a closed ideal in $L_{G}^{1}(\mathfrak{g})$, as is $\mathcal{K} \cap L^{1}(\mathfrak{g})$. 
Furthermore,

$$
L_{G}^{1}(\mathfrak{g}) /\left(\mathcal{K} \cap L_{G}^{1}(\mathfrak{g})\right)=\bigoplus_{\lambda \in \Lambda_{+}}\left(\mathbb{C} \mathcal{M}_{\lambda} \cap L_{G}^{1}(\mathfrak{g})\right) /\left(\mathcal{K} \cap L_{G}^{1}(\mathfrak{g})\right) .
$$

Proof. This theorem results easily from the basic fact ([4]) that $\Phi$ is an algebra homomorphism from $L_{G}^{1}(\mathfrak{g})$ onto $L_{G}^{1}(G)$ and that $L_{G}^{1}(G)$ may be decomposed via the group Fourier transform into ideals as

$$
L_{G}^{1}(G)=\bigoplus_{\lambda \in \Lambda_{+}} \mathbb{C} \chi_{\lambda}
$$

Given a $G$-modulator $m \in C(\mathfrak{g})$ such that $j m \in L_{G}^{1}(g)$, we obtain a character formula, as follows. Let $L_{m}: C(G) \rightarrow C(\mathfrak{g})$ be defined by $L_{m} \varphi=m \varphi^{\sim}$. Then by Proposition 2.4, $\Phi\left(L_{m} \varphi\right)$ is well-defined, and $\Phi\left(L_{m} \varphi\right)=\varphi$ for all $\varphi$.

It follows from the homomorphism property of $\Phi$ that for all $\varphi, \psi \in C_{G}(G)$,

$$
L_{m}(\varphi * \psi)=L_{m} \varphi * L_{m} \psi \quad(\bmod \mathcal{K}) .
$$

Now it is well-known that the dual of the commutative algebra $C_{G}(G)$ may be identified with the irreducible characters of $G$ by $f \rightarrow \int_{G} f(g) \chi(g) d g$. Similary the dual of $C(\mathfrak{g})$ may be identified, via the Fourier transform, with $\mathfrak{g}^{*}$, and $C_{G}(\mathfrak{g})^{\wedge}$ with the $G$-orbits in $\mathfrak{g}^{*}$ by $f \rightarrow \int_{\mathfrak{g}} \int_{\mathcal{O}_{\eta}} e^{i \beta X} d \mu_{\eta}(\beta) f(X) d X$. By Proposition (2.4) (i), such a homomorphism vanishes identically on $\mathcal{K}$ if and only if $\eta \in \Lambda$. For such an element $\lambda+\delta$, one expects that

$$
\int f(g) \chi_{\lambda}(g) d g=L_{m}^{*}(\lambda+\delta)(f)=\int_{\mathfrak{g}} \int_{\mathcal{O}_{\lambda+\delta}} e^{i \beta X} d \mu_{\lambda+\delta}(\beta) \nu(X) f^{\sim}(X) d X .
$$

This is established by the following result.

Proposition 2.7. Let $m \in L_{G}^{1}(\mathfrak{g}) \cap C_{G}(\mathfrak{g})$ be a modulator such that $j m \in L_{G}^{1}(\mathfrak{g})$. Then for all $f \in C_{G}^{\infty}(G)$

$$
\int f(g) \chi_{\lambda}(g) d g=\int_{\mathfrak{g}} \int_{\mathcal{O}_{\lambda+\delta}} e^{i \beta X} d \mu_{\lambda+\delta}(\beta) m(X) f^{\sim}(X) d X .
$$

Proof. From Kirillov's character formula, i.e. (2.1), the right hand side of the above equation equals

$$
\int_{\mathfrak{g}} j(X) \chi_{\lambda}^{\sim}(X) m(X) f^{\sim}(X) d X=\left\langle m, j\left(\chi_{\lambda} f\right)^{\sim}\right\rangle .
$$

Since $m$ is a modulator, this equals

$$
\int_{G} f(g) \chi_{\lambda}(g) d g
$$

The above formula will be called the character formula associated to $m$. If we choose a modulator $m \in \mathcal{E}_{G}(\mathfrak{g})$ we have a similar formula, viz.

$$
\int f(g) \chi_{\lambda}(g) d g=\left\langle m, j \chi_{\lambda}^{\sim} f^{\sim}\right\rangle
$$

To recover the usual Kirillov formula, one takes $m$ to be the characteristic function of a suitable neighbourhood $U$ of 0 in $\mathfrak{g}$ on which exp is a bijection and restricts $f$ to be in $C^{\infty}(\exp U)$. We may use the Kirillov formula to re-write the right hand side of the above expression as

$$
\left\langle\chi_{\lambda}, f\right\rangle=\left\langle m \mu_{\lambda+\delta}^{\vee}, f^{\sim}\right\rangle .
$$


In the next section, we will show that each modulator $M$ on the maximal torus has a natural extension $e(M)$ to $\mathfrak{g}$ such that $m=j e(M)$ is a $G$-modulator. The right hand side of the above expression then becomes

$$
\left\langle e(M) \mu_{\lambda+\delta}^{\vee}, j f^{\sim}\right\rangle=\left\langle\Phi\left(e(M) \mu_{\lambda+\delta}^{\vee}\right), f\right\rangle .
$$

The distributions $e(M) \mu_{\lambda+\delta}^{\vee}$ are our generalised characters.

\section{Modulators and the maximal torus}

(3.1) Introduction. In this section, we shall reduce the problem of finding modulators to one of abelian harmonic analysis, by reducing to the maximal torus. Firstly, we shall consider the case where $G$ actually is a torus.

(3.2) Modulators for tori. Let $T$ be a compact connected abelian Lie group, a torus; let $\mathfrak{t}$ be its Lie algebra and exp the exponential map. In this case, $\Gamma=\{H \in$ $\mathfrak{t}: \exp H=e\}$, and

$$
\Lambda=\left\{\lambda \in \mathfrak{t}^{*}:(\lambda, H) \in 2 \pi \mathbb{Z} \text { for all } H \in \Gamma\right\}
$$

may be identified with the set of characters of $T$.

We have the following characterization of $T$-modulators.

Proposition. Let $\mu$ be a distribution of compact support on $\mathfrak{t}$ or an element of $L^{1}(\mathfrak{t})$. The following conditions are equivalent.

(i) $\mu$ is a T-modulator.

(ii) $\sum_{\gamma \in \Gamma} \mu_{\gamma}$ converges to 1 in $\mathcal{D}^{\prime}$ (where $\mu_{\gamma}$ denotes $\mu$ translated by $\gamma$ ).

(iii) $\hat{\mu}(0)=1$ and $\hat{\mu}(\lambda)=0$ for all $\lambda \in \Lambda \backslash\{0\}$.

Proof. Let $f \in C^{\infty}(T)$. Then for $H \in \mathfrak{t}$ and $\gamma \in \Gamma, \tilde{f}(H+\gamma)=\tilde{f}(H)$.

Let $V \subseteq \mathfrak{t}$ be a fundamental domain for exp and let $\psi_{V}$ denote its characteristic function. Then

$$
\langle\mu, \tilde{f}\rangle=\sum_{\gamma \in \Gamma}\left\langle\psi_{V} \mu_{\gamma}, \tilde{f}\right\rangle=\left\langle\psi_{V} \sum_{\gamma \in \Gamma} \mu_{\gamma}, \tilde{f}\right\rangle .
$$

Thus we see that $\langle\mu, \tilde{f}\rangle=\langle 1, f\rangle$ if and only if $\psi_{V} \sum_{\gamma \in \Gamma} \mu_{\gamma}$ converges to 1 in $\mathcal{D}^{\prime}(V)$. Since $\sum_{\gamma \in \Gamma} \mu_{\gamma}$ is $\Gamma$-invariant and $V$ is a fundamental domain for $\Gamma$, this occurs if and only if $\sum_{\gamma \in \Gamma} \mu_{\gamma}$ converges to 1 in $\mathcal{D}^{\prime}$.

We have proved the equivalence of (i) and (ii).

To show the equivalence of (ii) and (iii), we use the Poisson summation formula on $\mathfrak{t}$ and $\mathfrak{t}^{*}$ with respect to the dual lattices $\Gamma$ and $\Lambda$, i.e.

$$
\sum_{\gamma \in \Gamma} f(\gamma)=\sum_{\lambda \in \Lambda} \hat{f}(\lambda) \quad \forall f \in \mathcal{D}
$$

If $\mu$ is a distribution of compact support or an element of $L^{1}(\mathfrak{t})$, and $f \in \mathcal{D}$, then using the above Poisson summation formula on $f_{X}$ (the translate of $f$ by $X$ ), we obtain

$$
\begin{aligned}
\left\langle\sum_{\gamma \in \Gamma} \mu_{\gamma}, f\right\rangle & =\left\langle\mu, \sum_{\gamma \in \Gamma} f_{\gamma}\right\rangle=\left\langle\mu, \sum_{\lambda \in \Lambda} \hat{f}(\lambda) e^{i \lambda(\cdot)}\right\rangle=\sum_{\lambda \in \Lambda} \hat{f}(\lambda)\left\langle\mu, e^{i \lambda(\cdot)}\right\rangle \\
& =\sum_{\lambda \in \Lambda} \hat{f}(\lambda) \hat{\mu}(\lambda) .
\end{aligned}
$$


From this we see that $\sum_{\gamma \in \Gamma} \mu_{\gamma} \equiv 1$ if and only if $\sum_{\lambda \in \Lambda} \hat{f}(\lambda) \hat{\mu}(\lambda)=\hat{f}(0) \forall f \in \mathcal{D}$ if and only if (iii) holds.

This proposition shows that $T$-modulators exist in abundance: for example, let $\mu$ be any Schwarz function whose Fourier transform satisfies (iii).

(3.3). In the case where $T$ is the maximal torus of a compact Lie group, we must take into account also the Weyl group actions on $\Gamma$ and $\Lambda$.

A straightforward corollary of the above result is

Corollary. Let $\mu$ be a $W$-invariant distribution of compact support on $\mathfrak{t}$ or an element of $L_{W}^{1}(\mathfrak{t})$. The following conditions are equivalent.

(i) $\mu$ is a T-modulator.

(ii) $\sum_{\gamma \in \Gamma} \mu_{\gamma}$ converges to 1 in $\mathcal{D}^{\prime}\left(\mathfrak{t}_{+}\right)$.

(iii) $\hat{\mu}(0)=1$, and $\hat{\mu}(\lambda)=0$ for all $\lambda \in \Lambda_{+} \backslash\{0\}$.

(3.4). We now consider a compact Lie group with maximal torus $T$ and define the extension of a distribution from $\mathfrak{t}$ to $\mathfrak{g}$.

Definition. Let $G$ be a compact connected Lie group with maximal torus $T$. Let $\mu \in \mathcal{D}^{\prime}(\mathfrak{t})$. We define $e(\mu) \in \mathcal{D}^{\prime}(\mathfrak{g})$ by

$$
\langle e(\mu), f\rangle=\frac{1}{|W|}\left\langle\mu,\left.\prod_{\alpha \in R_{+}} \alpha^{2} f^{G}\right|_{\mathfrak{t}}\right\rangle .
$$

It is easy to check that this does indeed define an element of $\mathcal{D}^{\prime}(\mathfrak{g})$. In fact, this is a compact version of the Harish-Chandra orbital integral map. The next lemma shows why it is a natural extension.

Lemma 3.5. For any distribution $\mu \in \mathcal{D}^{\prime}(\mathfrak{t}), e(\mu)$ is an Ad-invariant distribution on $\mathfrak{g}$. If $\mu$ is $W$-invariant of compact support, then $e(\mu)$ also has compact support, a G-invariant set such that

$$
\operatorname{supp}(e(\mu)) \cap \mathfrak{t}=\operatorname{supp} \mu .
$$

If $\mu \in L_{G}^{1}(\mathfrak{g})$, then

$$
e\left(\left.\mu\right|_{\mathfrak{t}}\right)(X)=\mu(X) \text { a.e. }
$$

Proof. Everything is obvious but the last sentence.

Let $f \in C_{c}^{\infty}(\mathfrak{g})$. Then

$$
\begin{aligned}
\left\langle e\left(\left.\mu\right|_{\mathfrak{t}}\right), f\right\rangle & =\frac{1}{|W|}\left\langle\left.\mu\right|_{\mathfrak{t}},\left.\prod_{\alpha \in R_{+}} \alpha^{2}(\cdot) f^{G}\right|_{\mathfrak{t}}\right\rangle \\
& =\frac{1}{|W|} \int_{\mathfrak{t}} \int_{G} f(g H) d g \mu(H) \prod_{\alpha \in R_{+}} \alpha^{2}(H) d H .
\end{aligned}
$$

By the Weyl integration formula on $\mathfrak{g}$, the latter equals

$$
\int_{\mathfrak{g}} \mu(X) f^{G}(X) d X=\langle\mu, f\rangle
$$

Thus $e\left(\left.\mu\right|_{\mathfrak{t}}\right)=\mu$ a.e.

It will be useful later to have a description of $e(\mu)^{\wedge}$ in terms of $\hat{\mu}$. 
Proposition 3.6. Suppose that $\mu$ is a distribution of compact support on $\mathfrak{t}$. Then $e(\mu)^{\wedge}$ is the distribution on $\mathfrak{g}^{*}$ defined by

$$
\left\langle e(\mu)^{\wedge}, \psi\right\rangle=\frac{C_{G}}{|W|}\left\langle\left(\prod_{\alpha \in R_{+}} i \alpha \mu\right)^{\wedge},\left.\psi^{G}\right|_{\mathfrak{t}^{*}}\right\rangle
$$

where $\psi \in C_{c}^{\infty}\left(\mathfrak{g}^{*}\right)$ and $\psi^{G}$ and $C_{G}$ are as defined in (2.1).

Proof. By definition,

$$
\left\langle e(\mu)^{\wedge}, \psi\right\rangle=\left\langle e(\mu), \psi^{\vee}\right\rangle=\frac{1}{|W|}\left\langle\mu,\left.\prod_{\alpha \in R_{+}} \alpha^{2}\left(\psi^{\vee}\right)^{G}\right|_{\mathfrak{t}}\right\rangle .
$$

Now for $H \in \mathfrak{t}$,

$$
\left(\psi^{\vee}\right)^{G}(H)=C_{G} \int_{G} \int_{\mathfrak{g}^{*}} \psi(\beta) e^{-i \eta(g H)} d \eta d g .
$$

Now we use the Weyl integration formula on $\mathfrak{g}^{*}$ to obtain, for $H \in \mathfrak{t}_{+}$,

$$
\begin{aligned}
\left(\psi^{\vee}\right)^{G}(H) & =C_{G} \int_{\mathfrak{t}_{+}^{*}} \int_{G} \psi(g \lambda) d g \int_{G} e^{-i g_{1} \lambda(H)} d g_{1} \prod_{\alpha \in R_{+}}\langle\alpha, \lambda\rangle^{2} d \lambda \\
& =C_{G} \int_{\mathfrak{t}_{+}^{*}} \psi^{G}(\lambda) \frac{A(-\lambda)(H)}{\prod_{\alpha \in R_{+}}(-i \alpha)(H)} d \lambda .
\end{aligned}
$$

(We have lost one factor of $\prod_{\alpha \in R_{+}}\langle\alpha, \lambda\rangle$ with Harish-Chandra's formula and the other by the definition of $\psi^{G}$ as the integral with respect to Liouville measure.)

Since $A(\lambda)$ is $W$-anti-symmetric, this equals

$$
\frac{C_{G}}{\prod_{\alpha \in R_{+}}(-i \alpha)(H)} \int_{\mathfrak{t}^{*}} \psi^{G}(\lambda) e^{-i \lambda(H)} d \lambda=\frac{C_{G}}{\prod_{\alpha \in R_{+}}(-i \alpha)(H)}\left(\left.\psi^{G}\right|_{\mathfrak{t}^{*}}\right)^{\vee}(H) .
$$

The formula now follows.

We now characterise $G$-modulators in terms of $T$-modulators. Firstly, let us consider distributions of compact support.

Proposition 3.7. Suppose that $\mu$ is a $W$-invariant distribution of compact support on $\mathrm{t}$.

The following are equivalent.

(i) $\mu$ is a $T$-modulator.

(ii) $j e(\mu)$ is a $G$-modulator.

Proof. Let $f \in C^{\infty}(G)$. Then by definition of the wrapping map $\Phi_{G}$ for $G$ and the extension $e(\mu)$, one has

$$
\left\langle\Phi_{G}(j e(\mu)), f\right\rangle=\left\langle e(\mu), j^{2} f^{\sim}\right\rangle=\frac{1}{|W|}\left\langle\mu,\left.j^{2} \prod_{\alpha \in R_{+}} \alpha^{2}\left(f^{G}\right)^{\sim}\right|_{\mathfrak{t}}\right\rangle .
$$

Now $j^{2} \prod_{\alpha \in R_{+}} \alpha^{2}=(\Delta \circ \exp )^{2}$. Thus our expression is equal to

$$
\frac{1}{|W|}\left\langle\mu,\left.\left(\Delta^{2} f^{G}\right)^{\sim}\right|_{\mathfrak{t}}\right\rangle=\frac{1}{|W|}\left\langle\Phi_{T}(\mu),\left.\Delta^{2} f^{G}\right|_{T}\right\rangle,
$$

where $\Phi_{T}$ denotes the wrapping map for $T$. 
Now $\mu$ is a $T$-modulator if and only if $\Phi_{T}(\mu)=1$, and this occurs if and only if, for all $f$, the right hand side of the above equality is $\frac{1}{|W|} \int_{T} f^{G}(t) \Delta(t)^{2} d t$, which, by the Weyl integration formula, equals $\int_{G} f(g) d g$.

Thus we see that $\Phi_{T}(\mu)=1$ if and only if $\Phi_{G}(j e(\mu))=1$.

We wish also to consider the case where $\mu$ might not have compact support. Let $L_{W}^{1}(\mathfrak{t})$ denote the $W$-invariant elements of $L^{1}(\mathfrak{t})$.

Corollary 3.8. Suppose $\mu \in L_{W}^{1}(\mathfrak{t})$. Then $j^{2} e(\mu) \in L^{1}(\mathfrak{g})$, and $\mu$ is a T-modulator if and only if $j e(\mu)$ is a $G$-modulator.

Proof. We saw in $(2.2)$ that $\Phi_{G}(j e(\mu))$ is well-defined if $j^{2} e(\mu)$ belongs to $L^{1}(\mathfrak{g})$.

Now

$$
\left\|j^{2} e(\mu)\right\|_{L^{1}(\mathfrak{g})}=\int_{\mathfrak{t}} \mu(H)|\Delta(\exp H)|^{2} d H
$$

Since $\Delta$ is a trigonometric polynomial, the integral on the right hand side is bounded for $\mu \in L^{1}(\mathfrak{t})$, so that $j^{2} e(\mu) \in L^{1}(\mathfrak{g})$, and the proof of the previous proposition applies.

We thus see that to find a $G$-modulator, it suffices to find a $T$-modulator $\mu$ (either in $L_{W}^{1}(\mathfrak{t})$ or a distribution of compact support) and to calculate $j e(\mu)$. Aside from questions of integrability, one obtains the most general $G$-modulator in this way.

Using the observation following 3.2 , we can now construct $G$-modulators from $T$-modulators which are $W$-invariant, and these can be found by simply taking a $W$-average of an arbitrary $T$-modulator.

We pause to calculate the Fourier tranform of $j e(\mu)$ in terms of the Fourier transform of $\mu$.

Proposition 3.9. Let $\mu$ be a $W$-invariant distribution of compact support on $\mathfrak{t}$ or an element of $L_{W}^{1}(\mathfrak{t})$. Then $(j e(\mu))^{\wedge}$ is an $\mathrm{Ad}^{*}$-invariant function on $\mathfrak{g}^{*}$ such that for $\beta \in \mathfrak{t}_{+}^{*}$

$$
(j e(\mu))^{\wedge}(\beta)=\frac{1}{\prod_{\alpha \in R_{+}} i\langle\alpha, \beta\rangle} \sum_{w \in W} \operatorname{sgn} w \hat{\mu}(w \beta+\delta) .
$$

Proof. Now

$$
\left\langle j e(\mu), e^{i \beta}\right\rangle=\frac{1}{|W|}\left\langle\mu, \prod_{\alpha \in R_{+}} \alpha^{2} j \int_{G} e^{i g \beta} d g\right\rangle .
$$

Using Harish-Chandra's formula for the Fourier transform of an orbit, the right hand side becomes

$$
\begin{aligned}
& \frac{1}{\prod_{\alpha \in R_{+}} i\langle\alpha, \beta\rangle} \frac{1}{|W|}\left\langle\mu, \Delta^{\sim} \sum_{w \in W} \operatorname{sgn} w e^{i w \beta}\right\rangle \\
& =\frac{1}{\prod_{\alpha \in R_{+}} i\langle\alpha, \beta\rangle} \sum_{w \in W} \operatorname{sgn} w\left\langle\mu, e^{i(w \beta+\delta)}\right\rangle
\end{aligned}
$$

since $\Delta^{\sim}=\sum_{w \in W} \operatorname{sgn} w e^{i w \delta}$ and $\mu$ is $W$-invariant. 
Note that $(j e(\mu))^{\wedge}$ is necessarily an analytic function, so the left hand side of the above formula is valid for all $\beta \in \mathfrak{t}^{*}$. The right hand side is also defined by a suitable application of L'Hôpital's rule when $\beta \in \mathfrak{t}^{*}$ is singular.

(3.10) Construction of a character. We now take up again the discussion at the end of $\S 2$. We showed there that if $m$ is a $G$-modulator, then we have the following character formula for $\chi_{\lambda}, \lambda \in \Lambda_{+}$. For $f \in C^{\infty}(G)$,

$$
\left\langle\chi_{\lambda}, f\right\rangle=\left\langle m \mu_{\lambda+\delta}^{\vee}, f^{\sim}\right\rangle .
$$

We now know that $m$ can be taken to have the form $j e(M)$, where $M$ is a $W$-invariant $T$-modulator which is a distribution of compact support on $\mathfrak{t}$ or an element of $L_{W}^{1}(\mathfrak{t})$. The above formula then reads

$$
\left\langle\chi_{\lambda}, f\right\rangle=\left\langle e(M) \mu_{\lambda+\delta}^{\vee}, j f^{\sim}\right\rangle
$$

from which we see that $\chi_{\lambda}=\Phi\left(e(M) \mu_{\lambda+\delta}^{\vee}\right)$.

Putting all the information we have together, we see that we have proved

Theorem. Let $M$ be a distribution of compact support or an integrable function on $\mathfrak{t}$ which is a $W$-invariant $T$-modulator, and let $m=j e(M)$. For $\lambda \in \Lambda_{+}$, define a distribution $C h_{\lambda}^{m}$ on $\mathfrak{g}$ by

$$
C h_{\lambda}^{m}=e(M) \mu_{\lambda+\delta}^{\vee}
$$

Then $\Phi\left(C h_{\lambda}^{m}\right)=\chi_{\lambda}$.

The distribution $\left(C h_{\lambda}^{m}\right)^{\wedge}=e(M)^{\wedge} * \mu_{\lambda+\delta}$ may be thought of as a fattened coadjoint orbit; it may be thought of as an 'electron cloud density' around the orbit $\mathcal{O}_{\lambda+\delta}$, or perhaps a 'quantum orbit'.

We now compute an explicit formula for $\left(C h_{\lambda}^{m}\right)^{\wedge}$ in terms of $\widehat{M}$.

Proposition 3.11. Let $m, M$ be as in Theorem 3.10. Then for $\psi \in C_{c}^{\infty}\left(\mathfrak{g}^{*}\right)$

$$
\left\langle\left(C h_{\lambda}^{m}\right)^{\wedge}, \psi\right\rangle=C_{G}\left\langle\widehat{M}(\lambda+\delta+\cdot),\left.\psi^{G}\right|_{\mathfrak{t} *}\right\rangle .
$$

Proof. By definition of the Fourier transform we have

$$
\left\langle\left(C h_{\lambda}^{m}\right)^{\wedge}, \psi\right\rangle=\left\langle e(M) \mu_{\lambda+\delta}^{\vee}, \psi^{\vee}\right\rangle=\left\langle e(M), \mu_{\lambda+\delta}^{\vee} \psi^{\vee}\right\rangle,
$$

Now we know from Harish-Chandra's formula that

$$
\mu_{\lambda+\delta}^{\vee}(H)=\frac{A(\lambda+\delta)(H)}{\prod_{\alpha \in R_{+}} i \alpha(H)},
$$

and that this function is Ad-invariant. In the proof of (3.6), it is shown that for $H \in \mathfrak{t}_{+}$

$$
\left(\psi^{\vee}\right)^{G}(H)=\frac{C_{G}}{\prod_{\alpha \in R_{+}}(-i \alpha)(H)}\left(\left.\psi^{G}\right|_{\mathfrak{t}^{*}}\right)^{\vee}(H) .
$$

Thus, by the definition of $e(M)$,

$$
\left\langle\left(C h_{\lambda}^{m}\right)^{\wedge}, \psi\right\rangle=\frac{C_{G}}{|W|}\left\langle M, A(\lambda+\delta)\left(\left.\psi^{G}\right|_{\mathfrak{t}^{*}}\right)^{\vee}\right\rangle .
$$

Since $M$ is $W$-invariant and $\left.\psi^{G}\right|_{\mathbf{t}^{*}}$ is $W$-anti-invariant, this expression is equal to

$$
C_{G}\left\langle e^{i(\lambda+\delta)} M, \psi^{G}||_{\mathfrak{t}^{*}}^{\vee}\right\rangle
$$

from which the result follows. 
This result says that, in order to find $\left(C h_{\lambda}^{m}\right)^{\wedge}$, one takes $\widehat{M}$, translates it by $\lambda+\delta$, and then symmetrizes by integrating with respect to Liouville measure around each coadjoint orbit. The result is multiplied by the constant $C_{G}$.

In the case when $\widehat{M}$ is a function on $\mathfrak{t}^{*}$, as happens if $M$ is a distribution of compact support, or an element of $L^{1}(\mathfrak{t})$, we have, at least formally, for $f \in C_{G}^{\infty}(G)$

$$
\begin{aligned}
\left\langle\chi_{\lambda}, f\right\rangle & =\left\langle C h_{\lambda}^{m}, j f^{\sim}\right\rangle=\left\langle\left(C h_{\lambda}^{m}\right)^{\wedge},\left(j f^{\sim}\right)^{\wedge}\right\rangle, \\
& =C_{G}\left\langle\widehat{M}(\lambda+\delta+\cdot),\left.\left(\left(j f^{\sim}\right)^{\wedge}\right)^{G}\right|_{\mathfrak{t}^{*}}(\cdot)\right\rangle \\
& =C_{G} \int_{\mathfrak{t}^{*}} \int_{\mathcal{O}_{\beta}}\left(j f^{\sim}\right)^{\wedge}(\eta) d \mu_{\beta}(\eta) \widehat{M}(\beta+\lambda+\delta) d \beta
\end{aligned}
$$

The reason why this formal calculation is not a priori valid is that $j f^{\sim}$ is not in general integrable, and so neither $\left(j f^{\sim}\right)^{\wedge}$ nor $\left.\left(j f^{\sim}\right)^{\wedge}\right|_{\mathfrak{t}^{*}}$ is well-defined. Proposition (3.13) below shows that we can make sense of $\left.\left(j f^{\sim}\right)^{\wedge}\right|_{\mathbf{t}^{*}}$ as a Radon measure on $\mathfrak{t}^{*}$ and $\left(j f^{\sim}\right)^{\wedge}$ as its Ad*-invariant extension to $\mathfrak{g}^{*}$. Thus, if $\widehat{M}$ is integrable with respect to this Radon measure (as will be the case for example if $\widehat{M}$ has compact support), the above chain of equalities is valid. First, a definition.

Definition 3.12. Let $f \in C_{G}^{\infty}(G)$. For $\lambda \in \Lambda_{+}$, denote by $c_{\lambda}$ the number

$$
\int_{G} f(x) \chi_{\lambda}(x) d x \text {. }
$$

Let $\gamma(f)$ be the Radon measure on $\mathfrak{t}_{+}^{*}$ defined by

$$
\gamma(f)=\sum_{\lambda \in \Lambda_{+}} d_{\lambda} c_{\lambda} \delta_{\lambda+\delta}
$$

where $\delta_{\lambda+\delta}$ is the Dirac measure at $\lambda+\delta \in \Lambda^{+}$. Further, let $\bar{\gamma}(f)$ be the Radon measure on $\mathfrak{g}^{*}$ defined by

$$
\bar{\gamma}(f)=\sum_{\lambda \in \Lambda^{+}} c_{\lambda} \mu_{\lambda+\delta}
$$

where, as usual, $\mu_{\lambda+\delta}$ is the Liouville measure on the orbit $\mathcal{O}_{\lambda+\delta}$. Note that for any $\operatorname{Ad}^{*}$-invariant $\varphi \in C_{c}^{\infty}\left(\mathfrak{g}^{*}\right),\langle\bar{\gamma}(f), \varphi\rangle=\left\langle\gamma(f),\left.\varphi\right|_{\mathfrak{t}^{*}}\right\rangle$.

Proposition 3.13. We have $\bar{\gamma}(f)=\left(j f^{\sim}\right)^{\wedge}$ in the sense that for any $\psi \in C_{c}^{\infty}(\mathfrak{g})$,

$$
\left\langle j f^{\sim}, \psi\right\rangle=\langle\bar{\gamma}(f), \hat{\psi}\rangle .
$$

Proof. Let $D$ be a $W$-invariant fundamental domain for exp $: \mathfrak{t} \rightarrow T$.

By the Weyl character formula and the Weyl integration formula, one has

$$
\begin{aligned}
c_{\lambda} & =\frac{1}{|W|} \int_{T} f(t) \chi_{\lambda}(t)|\Delta(t)|^{2} d t \\
& =\frac{1}{|W|} \sum_{w \in W} \operatorname{sgn} w \int_{D}(\Delta f)(\exp H) e^{i w(\lambda+\delta) H} d H \\
& =\int_{D}(\Delta f)(\exp H) e^{i(\lambda+\delta)(H)} d H \\
& =\left(\left.(\Delta f)^{\sim}\right|_{D}\right)^{\wedge}(\lambda+\delta) .
\end{aligned}
$$

Now, suppose that $\psi \in C_{c}^{\infty}(\mathfrak{g})$ is Ad-invariant. Then

$$
\left\langle j f^{\sim}, \psi\right\rangle=\int_{\mathfrak{g}} j(X) f(\exp X) \psi(X) d X
$$


and by the Weyl integration formula for $\mathfrak{g}$, this equals

$$
\begin{aligned}
\int_{\mathfrak{t}_{+}} j(H) f(\exp H) \psi(H) \prod_{\alpha \in R_{+}} \alpha(H)^{2} d H \\
\quad=\int_{\mathfrak{t}_{+}}(\Delta f)^{\sim}(H) \int_{\mathfrak{g}^{*}} \hat{\psi}(\eta) e^{i \eta H} d \eta \prod_{\alpha \in R_{+}} \alpha(H) d H
\end{aligned}
$$

We now use the dual Weyl formula on $\mathfrak{g}^{*}$ to see that this expression is equal to

$$
\left.\int_{\mathfrak{t}_{+}}(\Delta f)^{\sim}(H) \int_{\mathfrak{t}_{+}^{*}} \hat{\psi}\right|_{\mathfrak{t}}(\lambda) \int_{\mathcal{O}_{\lambda}} e^{i \beta H} d \mu_{\lambda}(\beta) \prod_{\alpha \in R_{+}}\langle\alpha, \lambda\rangle \prod_{\alpha \in R_{+}} \alpha(H) d \lambda d H
$$

By Harish-Chandra's formula we obtain

$$
\left.\sum_{w \in W} \operatorname{sgn} w \int_{\mathfrak{t}_{+}}(\Delta f)^{\sim}(H) \int_{\mathfrak{t}_{+}^{*}} \hat{\psi}\right|_{\mathfrak{t}}(\lambda) e^{i \lambda w H} \prod_{\alpha \in R_{+}}\langle\alpha, \lambda\rangle d \lambda d H .
$$

Now $f$ and $\left.\hat{\psi}\right|_{\mathfrak{t}}$ are $W$-invariant and $\Delta$ and $\prod_{\alpha \in R_{+}}\langle\alpha, \cdot\rangle$ and $W$-anti-invariant. Thus the above sum simplifies to

$$
\left.\frac{1}{|W|} \int_{\mathfrak{t}}(\Delta f)^{\sim}(H) \int_{\mathfrak{t}^{*}} \hat{\psi}\right|_{\mathfrak{t}}(\lambda) e^{i \lambda H} \prod_{\alpha \in R_{+}}\langle\alpha, \lambda\rangle d \lambda d H .
$$

By standard Poisson summation arguments on $\mathfrak{t},\left.(\Delta f)^{\sim}\right|_{\mathfrak{t}}$ is a periodic function whose $\mathfrak{t}$-Fourier tranform is a Radon measure on $\mathfrak{t}^{*}$ given by

$$
\left.\left((\Delta f)^{\sim}\right)\right|_{\mathfrak{t}} ^{\wedge}=\sum_{\lambda \in \Lambda}\left(\left.(\Delta f)^{\sim}\right|_{D}\right)^{\wedge}(\lambda) \delta_{\lambda}
$$

in the sense that for all $u \in \mathcal{S}(\mathfrak{t})$

$$
\left\langle\left.(\Delta f)^{\sim}\right|_{\mathfrak{t}}, u\right\rangle=\sum_{\lambda \in \Lambda}\left(\left.\Delta f^{\sim}\right|_{D}\right)^{\wedge}(\lambda) \hat{u}(\lambda) .
$$

Thus the above integral becomes

$$
\frac{1}{|W|} \sum_{\lambda \in \Lambda}\left(\left.\Delta f^{\sim}\right|_{D}\right)^{\wedge}(\lambda) \hat{\psi}(\lambda) \prod_{\alpha \in R_{+}}\langle\alpha, \lambda\rangle .
$$

Now by antisymmetry, if $\lambda \in \Lambda$ is fixed by some reflection in $W, \prod_{\alpha \in R_{+}}\langle\alpha, \lambda\rangle=0$. Furthermore, each element in $\Lambda$ not fixed by any reflection in $W$ is conjugate to one of the form $\lambda+\delta$, for some $\lambda \in \Lambda_{+}$, under a unique Weyl group element, and by Weyl's dimension formula $\prod_{\alpha \in R_{+}}\langle\alpha, \lambda+\delta\rangle=d_{\lambda}$. These considerations show that our sum simplifies to

$$
\sum_{\lambda \in \Lambda_{+}} d_{\lambda}\left(\left(\left.\Delta f^{\sim}\right|_{D}\right)^{\wedge}(\lambda+\delta) \hat{\psi}(\lambda+\delta)\right.
$$

Comparing this with (1), we see that

$$
\left\langle j f^{\sim}, \psi\right\rangle=\sum_{\lambda \in \Lambda_{+}} d_{\lambda} c_{\lambda} \hat{\psi}(\lambda+\delta) .
$$


If $\psi$ is an arbitrary, not necessarily Ad-invariant, function in $C_{c}^{\infty}(\mathfrak{g})$, the Adinvariance of $j$ and $f$ together implies that

$$
\left\langle j f^{\sim}, \psi\right\rangle=\left\langle j f^{\sim}, \psi^{G}\right\rangle .
$$

Furthermore, by our normalization of $\mu_{\lambda+\delta}$, we have

$$
d_{\lambda}\left(\psi^{G}\right)^{\wedge}(\lambda+\delta)=(\hat{\psi})^{G}(\lambda+\delta) .
$$

This proves the proposition.

\section{Decomposition of the Lebesgue measure on $\mathfrak{g}^{*}$}

(4.1) Introduction. Given that the Plancherel formula for $G$ may be written as

$$
f(e)=\sum_{\lambda \in \Lambda_{+}} d_{\lambda} \int f(g) \chi_{\lambda}(g) d g
$$

for every $f \in C^{\infty}(G)$, and the Plancherel formula for $\mathfrak{g}$ may be written as

$$
f(0)=\int_{\mathfrak{g}^{*}} \hat{f}(\beta) d \beta
$$

for $f \in C_{c}^{\infty}(\mathfrak{g})$, it is natural to look for a partition of the identity on $\mathfrak{g}^{*}$ of the form

$$
1=\sum_{\lambda \in \Lambda_{+}} d_{\lambda}\left(C h_{\lambda}^{m}\right)^{\wedge}+\sum_{i=1}^{\infty} k_{i}
$$

where $k_{i}^{\vee} \in \mathcal{K}^{\vee}$ and where $m=j e(M)$ for some suitably chosen $W$-invariant $T$ modulator $M$. One then has, formally at least,

$$
\Phi\left(1^{\vee}\right)=\sum_{\lambda \in \Lambda_{+}} d_{\lambda} \chi_{\lambda}
$$

This expresses Plancherel measure for $G$ as an image under $\Phi$ of the Plancherel measure for $\mathfrak{g}$, and provides an answer to the problem of unifying the construction of Plancherel measure for the compact and nilpotent cases.

The particular modulator chosen below belongs to $L^{1}(\mathfrak{t})$; its Fourier transform is continuous of compact support. It is the analogue of the Fejér kernel.

Definition 4.2. Let $\psi_{0}: \mathbb{R} \rightarrow \mathbb{R}$ be defined by

$$
\psi_{0}(x)=(1-|x|)_{+}= \begin{cases}1-|x| & \text { if }|x| \leq 1, \\ 0 & \text { if }|x|>1 .\end{cases}
$$

Let $\psi_{n}(x)=\psi_{0}(x-n)$, so that $\psi_{n}$ is supported on the interval $[n-1, n+1]$.

One verifies immediately that we may decompose the function $f(x)=x$ on $\mathbb{R}^{+}$ as follows:

$$
x=\sum_{n=0}^{\infty} n \psi_{n}(x) \quad \forall x \in \mathbb{R}^{+} .
$$

In fact, at most two terms in this sum are nonzero for any fixed $x \in \mathbb{R}^{+}$.

It follows that for every $\alpha \in R_{+}$,

$$
\langle\alpha, \beta\rangle=\sum_{n=0}^{\infty} n \psi_{n}(\langle\alpha, \beta\rangle) \quad \text { for all } \beta \in \mathfrak{t}_{+}^{*} .
$$


Hence, we may write

$$
\prod_{\alpha \in R_{+}}\langle\alpha, \beta\rangle=\prod_{\alpha \in R_{+}} \sum_{n=0}^{\infty} n \psi_{n}(\langle\alpha, \beta\rangle)=\sum_{\underline{n} \in \mathbb{N}^{q}} \prod_{\alpha \in R_{+}} n_{\alpha} \psi_{n_{\alpha}}(\langle\alpha, \beta\rangle)
$$

where we have used multi-index notation in the obvious fashion, and $q$ denotes card $R_{+}$.

This will be our basic decomposition. The next proposition analyses the properties of the functions $\prod_{\alpha \in R_{+}} \psi_{n_{\alpha}}(\langle\alpha, \cdot\rangle)$

Proposition 4.3. (i) Let $\beta \in \mathfrak{t}_{+}^{*}$. Then

$$
\prod_{\alpha \in R_{+}} \psi_{n_{\alpha}}(\langle\alpha, \beta\rangle)=0 \text { unless } n_{\alpha}-1<\langle\alpha, \beta\rangle<n_{\alpha}+1
$$

for all $\alpha \in R_{+}$.

(ii) Suppose $\lambda \in \Lambda$. Then

$$
\prod_{\alpha \in R_{+}} \psi_{n_{\alpha}}(\langle\alpha, \lambda\rangle)=0 \text { unless } n_{\alpha}=\langle\alpha, \lambda\rangle \text { for all } \alpha \in R_{+} .
$$

Proof. (i) is clear since $\psi_{n_{\alpha}}$ is supported on $\left[n_{\alpha}-1, n_{\alpha}+1\right]$.

(ii) If $\lambda \in \Lambda$, then $\langle\alpha, \lambda\rangle$ is an integer for all $\alpha \in R_{+}$. The only integer in $\left(n_{\alpha}-1, n_{\alpha}+1\right)$ being $n_{\alpha}$, the result is clear.

We thus see that there are two types of functions occurring in the sum (1). Firstly, for all $\lambda \in \Lambda_{+}$we have the function $\Psi_{\lambda}=\prod_{\alpha \in R_{+}} \psi_{\langle\alpha, \lambda+\delta\rangle}(\langle\alpha, \cdot\rangle)$, which is supported on the convex hull of the set $\{\lambda+\delta+w \delta: w \in W\}$. By the Weyl dimension formula, the coefficient $\prod_{\alpha \in R_{+}} n_{\alpha}$ of such a function in the sum (1) is exactly $d_{\lambda}$.

The second type of function that occurs in the sum is of the type

$$
k=\prod_{\alpha \in R_{+}} \psi_{n_{\alpha}}(\langle\alpha, \cdot\rangle)
$$

where there is no integral weight $\lambda$ with $\langle\alpha, \lambda+\delta\rangle=n_{\alpha}$ for all $\alpha \in R_{+}$. These functions have compact support and are zero at all points of $\Lambda_{+}$. By Proposition (2.4) (i), it follows that $k^{\vee} \in \mathcal{K}$ (i.e. $\Phi\left(k^{\vee}\right)=0$ ) for these functions.

We now proceed to construct an explicit modulator. Let $M$ be defined on $\mathfrak{t}$ by setting

$$
\widehat{M}(\beta)=\prod_{\alpha \in R_{+}} \psi_{0}(\langle\alpha, \beta\rangle)
$$

Since $\psi_{0}$ is continuous of compact support, $M$ is well-defined by this formula as a $W$-invariant function on $\mathfrak{t}$. Indeed, $M$ is defined by

$$
M(H)=\int_{\mathfrak{t}^{*}} \prod_{\alpha \in R_{+}} \psi_{0}(\langle\alpha, \beta\rangle) e^{i \beta(H)} d \beta,
$$

and by elementary Fourier analysis

$$
M(H)=\underset{\alpha \in R_{+}}{*}\left(\frac{\sin \alpha(H)}{\alpha(H)}\right)^{2},
$$


which is a $W$-invariant element of $L^{1}(\mathfrak{t})$. Furthermore, by our construction $\hat{\mu}(0)=1$ and $\widehat{M}$ vanishes on all other points of $\Lambda$.

Proposition 4.4. We may write

$$
\prod_{\alpha \in R_{+}}\langle\alpha, \beta\rangle=\sum_{\lambda \in \Lambda_{+}} d_{\lambda} \widehat{M}(\beta-(\lambda+\delta))+\sum_{\underline{n} \in \mathbb{N} q} k_{\underline{n}},
$$

where $\Phi\left(k_{\underline{n}}^{\vee}\right)=0$ for each function $k_{\underline{n}}$.

Proof. The terms in (1) of the second type correspond to the second sum in our statement. The terms in (1) of the first type have the form

$$
\begin{gathered}
\prod_{\alpha \in R_{+}} \psi_{\langle\alpha, \lambda+\delta\rangle}(\langle\alpha, \beta\rangle)=\prod_{\alpha \in R_{+}} \psi_{0}(\langle\alpha, \beta-\lambda-\delta\rangle) \\
=\widehat{M}(\beta-(\lambda+\delta)\rangle . \quad \square
\end{gathered}
$$

To see that this constitutes a decomposition of Lebesgue measure, suppose that $\psi \in C_{c}^{\infty}\left(\mathfrak{g}^{*}\right)$. Then by the dual Weyl integration formula

$$
\int_{\mathfrak{g}^{*}} \psi(\eta) d \eta=\int_{\mathfrak{t}_{+}^{*}} \psi^{G}(\beta) \prod_{\alpha \in R_{+}}\langle\alpha, \beta\rangle d \beta .
$$

Applying the formula of Proposition (4.4), one obtains

$$
\begin{aligned}
\int_{\mathfrak{g}^{*}} \psi(\eta) d \eta & =\sum_{\lambda \in \Lambda_{+}} d_{\lambda} \int_{\mathfrak{t}_{+}^{*}} \psi^{G}(\beta) \widehat{M}(\beta-(\lambda+\delta)) d \beta \\
& +\sum_{\underline{n} \in \mathbb{N}^{q}} \int_{\mathfrak{t}_{+}^{*}} k_{\underline{n}}(\beta) \psi^{G}(\beta) d \beta .
\end{aligned}
$$

If $\psi=\left(j f^{\sim}\right)^{\wedge}$ for some $f \in C^{\infty}(G)$, then the second sum vanishes, and by the discussion following (3.11) and (3.13), the first sum becomes

$$
\frac{1}{C_{G}} \sum_{\lambda \in \wedge_{+}}\left\langle\left(C h_{\lambda}^{m}\right)^{\wedge}, \psi\right\rangle=\frac{1}{C_{G}} \sum_{\lambda \in \Lambda_{+}} d_{\lambda}\left\langle\chi_{\lambda}, f\right\rangle .
$$

This is the desired relationship between the Lebesgue measure on $\mathfrak{g}^{*}$ and the Plancherel measure on $\hat{G}$.

\section{An eXtension of THE CONCEPT OF MODUlator}

(5.1). For certain of the applications we have in mind it is desirable to use $T$ modulators $M$ where $\widehat{M}$ has compact support but $M$ is not an element of $L^{1}(\mathfrak{t})$. For example, we may wish to take $\widehat{M}$ to be a characteristic function of some $W$ invariant neighbourhood of 0 which contains no nonzero elements of $\Lambda$. In this case, we may still define $M$ as a distribution acting in a principal value sense, and the preceding analysis is still valid. It is the purpose of this section to briefly sketch the technicalities involved. 
Definition 5.2. Let $\mathbb{1}_{S}$ denote the characteristic function of a set $S$. Suppose that $m$ is a locally integrable function on $\mathfrak{g}$ such that for any Ad-invariant connected neighbourhood $V$ of $0, \lim _{n \rightarrow \infty}\left\langle\mathbb{1}_{n V} m, j f^{\sim}\right\rangle$ exists and has the same value. We shall say that $m$ is an (extended) $G$-modulator, and denote the common value of this limit by p.v. $\int_{\mathfrak{t}} m(X) j(X) \tilde{f}(X) d X=\langle\Phi m, f\rangle$.

Clearly, $\Phi m$ is a distribution on $G$, which is central whenever $m$ is Ad-invariant. Specializing to $\mathfrak{t}$, we obtain the notion of an extended $T$-modulator.

Proposition (3.2) and Corollary (3.3) continue to hold in this setting. If we define the extension of a $W$-invariant extended $T$-modulator as in (3.4), Lemma (3.5) is still valid, as is Proposition (3.7).

We shall say that an extended $G$-modulator $m$ has a Fourier transform if for all $\beta \in \mathfrak{g}^{*}$, for every Ad-invariant connected neighbourhood $V$ of the origin in $\mathfrak{g}$, the limit

$$
\lim _{n \rightarrow \infty} \int_{n V} m(X) e^{i \beta X} d X
$$

exists and has the same value.

The common value is denoted by $\widehat{M}(\beta)$.

With this notation, if $M$ has a Fourier transform on $\mathfrak{t}$, then $e(M)$ has a Fourier transform on $\mathfrak{g}$, and if we define $C h_{\lambda}^{m}$ as before, the analogue of Proposition (3.11) holds. Furthermore, in the case where $\widehat{M}$ is a function of compact support, we have the formula

$$
\left\langle\chi_{\lambda}, f\right\rangle=\left\langle\widehat{M}(\cdot+\lambda+\delta),\left.\left(j f^{\sim}\right)^{\wedge}\right|_{\mathbf{t}^{*}}(\cdot)\right\rangle,
$$

where the right hand side is a pairing between a Radon measure and a translate of $\widehat{M}$.

An example of this kind of extended modulator is obtained as follows. Let $\left\{\lambda_{1}, \cdots, \lambda_{\ell}\right\}$ be a set of fundamental dominant weights for $\Lambda$.

Definition 5.4. We shall say that $A \subset \mathfrak{t}^{*}$ is a $W$-fundamental domain for $\Lambda$ if $A$ is a convex $W$-invariant set which is in addition a fundamental domain for $\Lambda$.

Lemma. There exists a unique $W$-fundamental domain $A$ in $\mathbf{t}^{*}$.

Proof. A may be constructed as follows. Let

$$
A_{i}=\left\{\eta \in \mathfrak{t}^{*}: \frac{-\left|\lambda_{i}\right|^{2}}{2} \leq\left(\eta, \lambda_{i}\right) \leq \frac{\left|\lambda_{i}\right|^{2}}{2}\right\}
$$

and let $A$ be the intersection of all the $A_{i}$ and their $W$-conjugates.

It is easy to see that the $\Lambda_{+}$-translates of $A$ tessellate a simply connected region in $\mathfrak{t}^{*}$ containing all of $\mathfrak{t}_{+}^{*}$. This is a canonical tessellation. Furthermore, if we let $M=\left(\mathbb{1}_{A}\right)^{\vee}$, then we have the character formula

$$
\int_{G} f(x) \chi_{\lambda}(x) d x=\left\langle C h_{\lambda}^{m}, j f^{\sim}\right\rangle .
$$

However, the characters $C h_{\lambda}^{m}$ do not give such a nice decomposition of Lebesgue measure as was found in the last section. 


\section{REMARKS ON APPLICATIONS TO MULTIPLIERS}

Many theorems have been proved about central $L^{p}$ and $L^{p}-L^{q}$ multipliers on compact groups, by using Weyl's integration formula to transfer estimates on $G$ to estimates on the maximal torus $T$ (e.g. [2]). This has involved studying the integrability of negative powers of the Weyl function $\Delta$. Since $\Delta$ vanishes at the origin, this creates many technical problems.

We may now propose an alternative strategy; that is, by use of an appropriate modulator, to transfer the estimates to the Lie algebra $\mathfrak{g}$. The negative powers of $\Delta$ are then replaced by negative powers of $j$, which is smooth and nonzero at the origin.

We shall further develop these ideas in future work.

\section{REFERENCES}

1. N. Berline and M. Vergne, Fourier transforms of orbits of the coadjoint representation, in Representation theory of reductive groups, Birkhäuser, 1983, pp 53-67. MR 85g:22026

2. R. Coifman and G. Weiss, Transference methods in analysis, CBMS lecture notes, Vol. 31, Amer. Math. Soc., Providence, RI, 1977. MR 58:2019

3. A. H. Dooley, J. Repka, and N. J. Wildberger Sums of adjoint orbits, Lin. Multilin. Alg. 36 (1993), 79-101. MR 95k:22025

4. A. H. Dooley and N. J. Wildberger, Harmonic analysis and the global exponential map for compact Lie groups, Funktsional. Anal. i Prilozhen. 27 (1993), no. 1, 25-32; English transl., Funct. Anal. Appl. 27 (1993), 21-27. MR 94e:22032

5. A. H. Dooley and F. Ricci, On the structure of the G-invariant Fourier algebra, Boll. Un. Mat. Ital. A (7) 9 (1995), 37-45. MR 96d:43011

6. M. Duflo, Opérateurs différentiels bi-invariants sur un groupe de Lie, Ann. Sci. Ecole Norm. Sup. 10 (1977), 265-288. MR 56:3188

7. F. Ricci and G. Travaglini, $L^{p}-L^{q}$ estimates for orbital measures and Radon transforms on compact Lie groups and Lie algebras, J. Funct. Anal. 129 (1995), 132-147. MR 96c:22016

8. N. R. Wallach, Symplectic geometry and Fourier analysis, Math. Sci. Press, Brookline, MA, 1977. MR 58:7715

9. N. J. Wildberger, Hypergroups and harmonic analysis, Proc. Centre Math. Anal. (ANU) 29 (1992), 238-253. MR 93j:43013

School of Mathematics, The University of New South Wales, Sydney 2052, Australia 